\title{
Application of IRF-Kriging to the mapping of environmental noise generated by quarrying plants
}

\author{
G. Alfaro Degan, D. Lippiello \& M. Pinzari \\ Department of Engineering, ROMA TRE University, Italy
}

\begin{abstract}
A geostatistical solution is presented to assess the acoustic impact of a quarry plant near Rome by drawing acoustic maps all around the selected site. The method utilised to determine the sound pressure level in the area of interest is the Intrinsic Random Function (IRF) Kriging with external drift. This technique allows the value of a regionalized variable to be estimated at a point in which it is unknown through the use of two sets of data. The first one is obtained by carrying out phonometric samples at various points near the site, while the second one is from a deterministic model of sound propagation defined in accordance with norms in force.

In order to test the quality of the estimates and especially the contribution of the external drift, acoustic maps on a local scale are drawn up both with and without drift. The quality of the results obtained is tested by means of cross validation.

The experimental results demonstrate that, providing it is strongly correlated to the first, the introduction of this secondary information, in a stochastic framework of reference, contributes to a more efficient representation of the spatial variability of sound propagation, and thus to an improvement in the quality of the correlated estimate. In the case study analysed here the introduction of drift permits a reduction of estimate variance, for the same number of available measurements. Again from the cross validation data and estimate variance it was observed that the use of external drift allows loss of information (e.g. due to a reduction in the monitored network) to be compensated for without invalidating the quality of the estimate itself.

Keywords: noise assessment, environmental impact, IRF Kriging with external drift, quarries.
\end{abstract}




\section{Introduction}

The evaluation of the acoustic impact on the environment constitutes an important step towards examining the environmental compatibility of the extraction site with the surrounding area, both during the plant planning phase and during the urban planning of the neighbouring areas. In this regard the EU directive and the relative Italian legislation (Italian Decree 14/11/1997) require a preliminary evaluation of the acoustic impact during the planning stage of the site and periodical checks thereafter to ensure that the established standards are upheld throughout the construction of the site as well as during the operation and development of the plant. Obviously this approach has the effect to safeguard also the acoustic climate into the quarry itself so representing an important aspect regarding health and safety management for those involved in quarry operations [1]. There have been numerous studies which have examined the problem both with regard to the planning stage, with various different approaches [2-4], and with regard to evaluation during plant operation. One common approach, also applied to other physical agents in quarry environment, [5], consists in monitoring the parameters in the field and successively modelling the phenomenon of propagation by means of algorithms suggested by the standard and refined to suit the specific case in question. Thus the standard ISO 9613-2, constitutes a starting point for later refinements and adjustments. This standard proposes a source model which relies upon a detailed characterisation of the active sound sources, as well as on later measurements in order to quantify the attenuating factors due to acoustic dispersion and absorption, [6]. As a result of the numerous active sources on site of quarrying and stonework machinery, the monitoring campaigns to determine the nature of the phenomenon may become onerous in terms of quantity and time, [7]. This is especially true with regard to sites in which ornamental stone is extracted (the category to which the tuff in our case study belongs) where there are also many mobile sources due to which the propagation model must undergo a considerable degree of further elaboration, [8].

In this article the phenomenon of sound propagation is interpreted in a stochastic context. The sound pressure level is thus considered as a regionalized variable and its spatial distribution is generated by a stochastic process [9]. The technique utilised here, which is widely used in environmental science, is that of Kriging with external drift introduced by the Matheron group in some application studies $[9,10]$, until more recent applications $[11,12]$. This technique allows the value of a regionalized variable to be estimated at a point in which it is unknown through the use of both its monitored data and data from an auxiliary variable, correlated to the primary and known throughout the dominion. Recent studies, applied in various fields of environmental science, have demonstrated that the use of this auxiliary variable improves the quality of the estimate, $[13,14]$. The objective of this article is to demonstrate, with the aid of a case study, that the sound monitoring network of an area adjoining an industrial site, may be considerably reduced without causing a reduction in the quality of the estimate of the acoustic climate, provided that an auxiliary variable is introduced which is correlated to the principle variable and is able to integrate the information loss. 


\section{Materials and methods}

The work was organised as follows. The first step constitutes a measurement campaign conducted using 30 receptors located in the area adjacent to the extraction site. Each measurement lasted 30 minutes in order to guarantee that they were representative of typical conditions so that any events that may disturb the measurements might be determined (e.g. traffic or other manmade noise from nearby sources). Subsequently, using the source model derived from ISO 9613 described in detail below, and the machinery manufacturers' noise output specifications, as well as geomorphological information about the site, an evaluation of the noise impact on the environment was carried out.

The evaluation considers a square-shaped area, with sides measuring 310 metres, in which the quarry plant is located at its centre. This domain area is divided into a regular grid with 700 nodes spaced at intervals of 12 metres. Then, by means of a code, the Intrinsic Kriging with external drift estimation system is implemented, in which the primary variable consists of a set of sound pressure level values obtained through monitoring, whilst the secondary variable is another set of sound pressure level values which this time, however, are calculated from the source model. In order to guarantee maximum simplicity in the development of the model, the entire plant is modelled starting from the contribution of the sources present whose combined effect at a given point of the domain is calculated as the sum of the sound energy from the sources which are simultaneously active on site. The estimate is carried out in the 700 nodes of the grid defined above and, for a more immediate visual representation, the isobels are plotted providing an acoustic map of the dominion. In order to validate the results, four distinct scenarios are proposed which vary according to the number of measurements utilised and whether or not external drifting is used as auxiliary information. Initially acoustic maps are obtained using only half of the data available from the monitoring network (i.e. only 15 measurements) with or without drift. Subsequently, the same comparison is made using all 30 of the available data. Each scenario is verified experimentally by means of validation, cross validation, or both.

\subsection{Field study and data sampling}

The area analysed is located to the north of Rome, near the Municipality of Riano, which is an important industrial area in Lazio where Tuff is extracted.

The final product consists standard sized blocks for use in the building trade. The mineral deposit is divided into various areas and the rock is divided and is extracted by means of vertical cutting using the stone cutter followed by the block removing machine which cuts horizontally as well as crosswise, separating the blocks and allowing them to be removed from the deposit.

The monitoring network permitted the sampling of the Equivalent Continuous Sound Pressure Level (Leq) across the 30 nodes of an irregular grid all around the area shown in figure 1 below. Each location was observed for a period of thirty 
minutes. The aim of each survey was that of characterizing the acoustic climate due to quarry plant operations and therefore any noise from other sources was filtered out

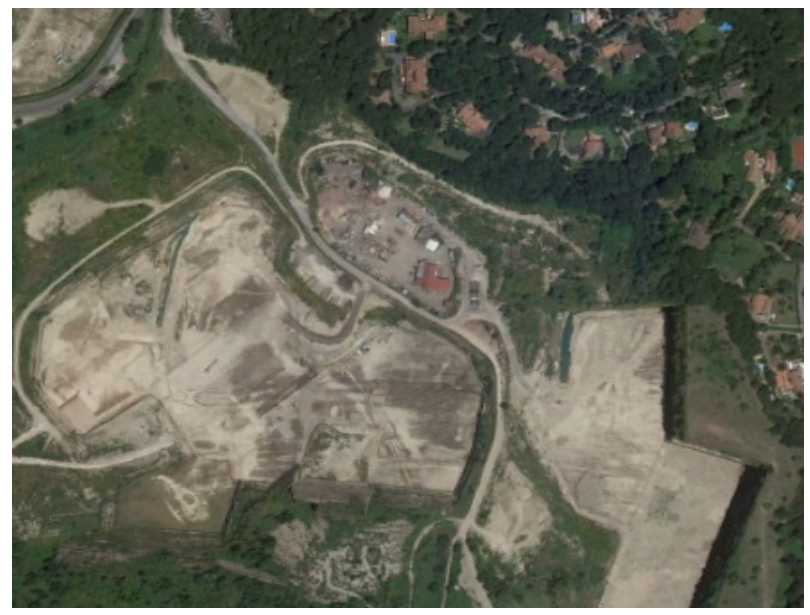

Figure 1: The selected area.

In each of the 30 receptors selected in the domain, the $\mathrm{L}_{\mathrm{A} \text {,eq, }}$ Te expressed in $(\mathrm{dB}(\mathrm{A}))$ is given by

$$
L_{A, e q, T e}=10 \log _{10} \frac{1}{T_{0}} \int_{0}^{T_{0}} \frac{\mathrm{p}_{\mathrm{A}}(\mathrm{t})^{2}}{\mathrm{p}_{0}^{2}} \mathrm{dt}
$$

where $T_{0}$ represents the observation time equal to 30 minutes; $p_{0}$ the sound pressure level reference value equal to $20 \mu \mathrm{Pa} ; \mathrm{p}_{\mathrm{A}}(\mathrm{t})$ the sound pressure level, weighted according to curve A, due to the active sources during the observation interval $(\mathrm{Pa})$.

\subsection{Estimation method: Intrinsic Kriging with external drift}

According to a probabilistic approach to modelling the physical mechanism of sound propagation, the $\mathrm{n}$ observations $\mathrm{z}(\mathrm{x})$ are interpreted as a realization of a random function $Z(x)$ at location $x$. In this framework Intrinsic Kriging with external drift is the method used to estimate the Sound Pressure Level (SPL) in the selected area. This approach allows the estimate of a random primary variable $\mathrm{Z}(\mathrm{x})$ known only at a small set of points in the domain while taking into account not only the $\mathrm{n}$ available data of the primary variable itself, but also considering another variable $\mathrm{s}(\mathrm{x})$ exhaustively known in the same domain. As variable $\mathrm{s}(\mathrm{x})$ should be strongly correlated with the primary variable $\mathrm{Z}(\mathrm{x})$, the former may be used to provide additional information regarding the spatial structure of the latter.

Considering a linear estimator we may obtain the value of the variable as a linear combination of $\mathrm{N}$ data values

$$
\tilde{z}\left(x_{0}\right)=\sum_{i}^{N} \lambda_{i} z\left(x_{i}\right)
$$


in which $\lambda_{i}$ coefficients are to be determined, together with $\mu_{i}$ Lagrange multipliers, as results of the following Kriging system:

$$
\sum_{\beta}^{N} \lambda_{\beta} K\left(x_{\alpha}-x_{\beta}\right)+\sum_{l} \mu_{l} f^{l}\left(x_{\alpha}\right)+\sum_{i} \mu_{i} s\left(x_{\alpha}\right)=K\left(x_{\alpha}-x_{0}\right)
$$

whatever $\alpha$ is

$$
\begin{gathered}
\sum_{\beta}^{N} \lambda_{\beta} f^{l}\left(x_{\beta}\right)=f^{l}\left(x_{0}\right) \\
\sum_{\beta}^{N} \lambda_{\beta} s\left(x_{\beta}\right)=s\left(x_{0}\right)
\end{gathered}
$$

The three equations are respectively imposed to ensure an unbiased estimation for primary variable and secondary one, (4) (5), while eqn. (3) is the optimization condition that implies the minimization of estimation variance.

In particular, dealing with a non-stationary phenomenon the conditional expectation of the primary variable may be expressed as a deterministic trend function:

$$
E[Z(x)]=m(x)
$$

It varies smoothly at local scale and it is expressed as:

$$
E[z(x)]=\sum_{l=0}^{L} a_{l} f^{l}(x)
$$

where the $\mathrm{f}^{l}$ functions are all the monomials of degree $\leq \mathrm{k}$ (degree of the drift) and the $\mathrm{a}_{l}$ 's are unknown coefficients. The total number of monomials, denoted $\mathrm{L}$ +1 , is equal to $(\mathrm{K}+1)(\mathrm{K}+2) / 2$ in $\mathrm{R}^{2}$.

In many cases the spatial variable exhibits a trend that is not always simple or easy to interpret and, in such cases, the theory of intrinsic random functions of order $\mathrm{k}$ (IRF-k) has proven to be an efficient methodology.

The basic idea by Matheron [15], is that, instead of working with the experimental data, one works with combinations of the experimental data that filter out any drift in the same way that finite differences filter a trend in time series analysis. Such linear combinations are called Allowable Linear Combinations (ALC) or generalized increments of order $\mathrm{k}$ when they filter out a drift up to order $\mathrm{k}$. From an applied point of view the most interesting aspect of IRF-k theory is that the generalized covariance of order $k$ characterizes an IRF-k in the same sense that a covariance function characterizes a stationary random function.

Thus dealing with non-stationary cases, means the $\mathrm{K}(\mathrm{h})$ function may not be defined for a given $\mathrm{h}$ distance by taking pairs of sampled points. Under these conditions, the generalized covariance is determined as the result of an automatic fitting process in which the Generalized Covariance (GC) is expressed as a given a priori model whose coefficients are chosen according to the Minimum Norm Quadratic Unbiased Estimation (MINQUE) method, [16].

In particular the Generalized Covariance is expressed as:

$$
K(h)=C_{0}+\sum_{m=0}^{K}(-1)^{m+1} b_{2 m+1}|h|^{2 m+1}+\sum_{m=1}^{K}(-1)^{m+1} b_{2 m}|h|^{2 m} \log h
$$

in which the three parameters $C_{0}, b_{2 m+1}, b_{2 m}$ are not negative and assessed using the minimum norm quadratic unbiased estimation method.

For the purpose, some calculus computer code is developed and run. 
In practice once a $\mathrm{K}$ order is assigned, the program allows one to define the set of coefficients minimizing the norm of the matrix system, thus providing unbiased estimate.

The only notable specification refers to the variable itself: since the estimation method is based on a linear interpolation of values as shown in eqn. (2), the whole procedure is developed considering the regionalized variable as sound pressure $\left(\mathrm{p}_{\mathrm{a}}\right)$ and only once the estimation is carried out, the conversion from sound pressure to its corresponding level is carried out.

\subsection{The outdoor sound propagation model}

The secondary variable covering the full domain on a regular grid was identified in Sound Pressure then converted into its corresponding level (SPL), from a deterministic propagation model.

The proposed model calculates the sound pressure level at the receptors, starting from the noise output data of the active sources, whilst considering the dispersion and reflection effects of the signal in the propagation phase. From standard ISO 9613-2 (1996) Attenuation of Sound During Propagation Outdoors, it is:

$$
L_{S, A, f}=L_{W, A}+D_{I}+K_{0}-D_{S}-A
$$

where: $L_{S, A, f}$ is the predicted sound pressure level for the single frequency $\mathrm{f}$ and at a certain distance from the sound source expressed in $\mathrm{dB}(\mathrm{A}) ; D_{I}$ is the directivity index of the source $(\mathrm{dB}(\mathrm{A}))$; $\mathrm{L}_{\mathrm{W}, \mathrm{A}, \mathrm{i}}$ is the sound power level of the source given by the machine manufactures $(\mathrm{dB}(\mathrm{A})) ; \mathrm{K}_{0}$ is the shape factor relative to the emission characteristics of the machine $(\mathrm{dB}(\mathrm{A})) ; \mathrm{D}_{\mathrm{s}}$ is the geometric divergence term expressed in $\mathrm{dB}(\mathrm{A})$ and depending on distance from source to the receptor.

The term A represents the set of factors which contribute to the absorption of sound energy $(\mathrm{dB}(\mathrm{A}))$.

In the case study presented here, the entire plant is modelled starting from the contribution of the four sources present whose combined effect at a given point of the domain is calculated as the sum of the sound energy from the sources which are simultaneously active on site. So the sound level at a given point outside the plant is:

$$
L_{P A, E}=10 \log \left(\sum_{i}^{N} 10^{0.1} L_{P, A, i}\right)
$$

where: $L_{P, A, E}$ is the sound pressure level at a given point $(\mathrm{dB}(\mathrm{A}))$; $\mathrm{N}$ represents the number of active sources; $L_{P a i}$ represents the sound pressure level at a given point generated by the i-th source obtained starting from eqn. (9) and expressed in $(\mathrm{dB}(\mathrm{A}))$.

\section{Data and results}

Table 1 below indicates the machinery considered in the case study with the relative noise emission specifications. These levels along with the data relative to the attenuation of the signal constitute the input data of the model. 
Table 1: Model, type and sound power level for each modeled sound source.

\begin{tabular}{|c|c|c|}
\hline Machine Type & Model & Lw (dB(A)) \\
\hline Vertical cutter (liner) & Pavoni CV 300 & 118.0 \\
\hline Block remover & Pavoni CV 180 & 119.0 \\
\hline Dumper & Volvo A25C & 112.0 \\
\hline Wheel loader & Volvo L70D & 106.0 \\
\hline
\end{tabular}

The forecast is shown in figure 2 by means of a SPL map showing the isobels. This is constructed from the data obtained from a regular grid with nodes spaced $12 \mathrm{~m}$ apart in a square-shaped domain with sides of about $300 \mathrm{~m}$ long.
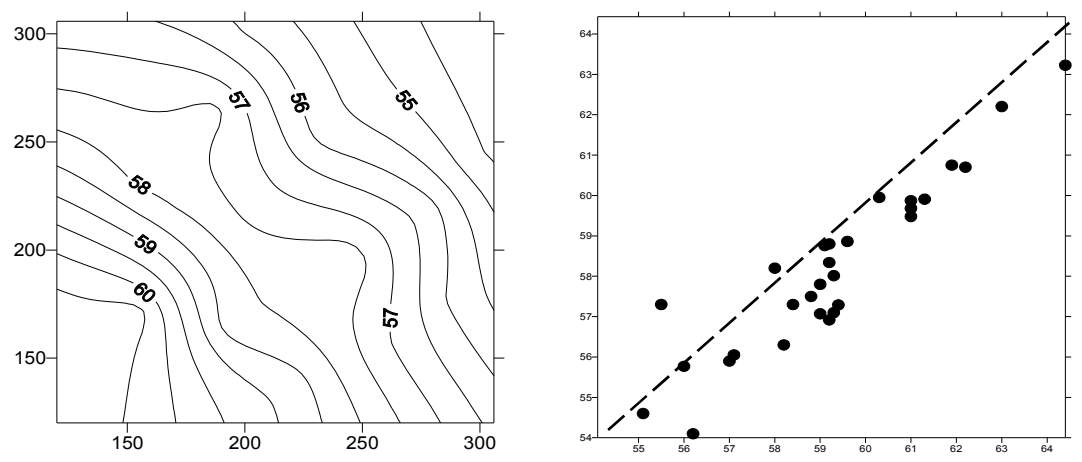

Figure 2: ISO 9613 SPL map indicating the isobels of the area and the associated scatterplot.

For quick reference, on the right of the same figure 2, a scatterplot is given between the equivalent sound pressure level values found at the points sampled (abscissa) and the corresponding values obtained from the model (ordinate) both expressed in $\mathrm{dB}(\mathrm{A})$.

In order to employ the estimate using IRF Kriging, an ad hoc algorithm was utilised which could calculate the estimate from a grid of defined points, starting from the available dataset. A further option of external drift allowed auxiliary information to be taken into consideration.

To implement the estimate two scenarios were considered: the first with 15 survey measurements, chosen at random from among the 30 measurements available; and the second which utilised all 30 measurements. Each input dataset was then considered both with and without external drift. Each result is indicated with its relative SPL map in the selected square shaped area with side measuring 310 meters, showing the isobels in $\mathrm{dB}(\mathrm{A})$. The quality of the result obtained is tested by means of validation and represented by scatterplots between the equivalent sound pressure level values found at the points sampled (abscissa) and the corresponding values obtained from IRF Kriging System (ordinate) both expressed in $\mathrm{dB}(\mathrm{A})$ as shown in figures 4 and 6 . 

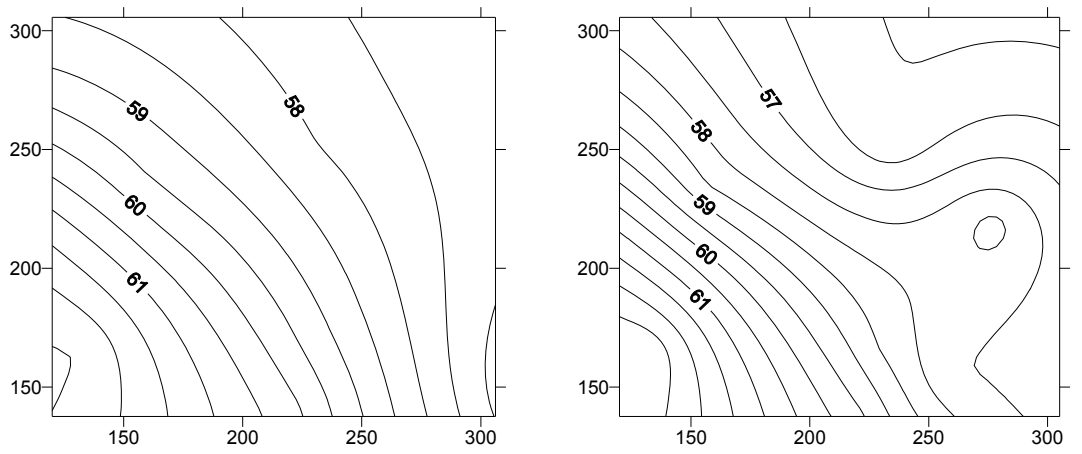

Figure 3: SPL maps in the domain (15 samples input without external drift on the left and with drift on the right).
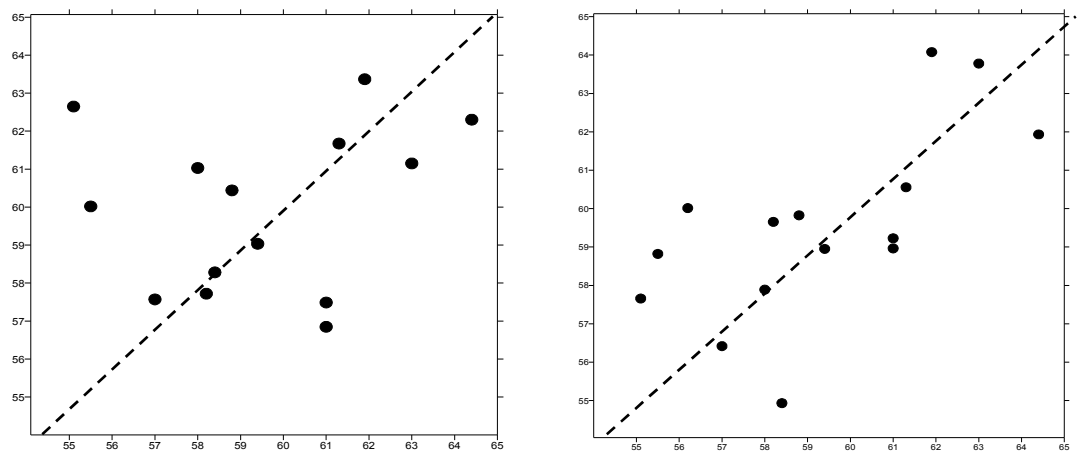

Figure 4: Scatterplot sampled values vs estimated (15 samples input without drift) expressed in $\mathrm{dB}(\mathrm{A})$.
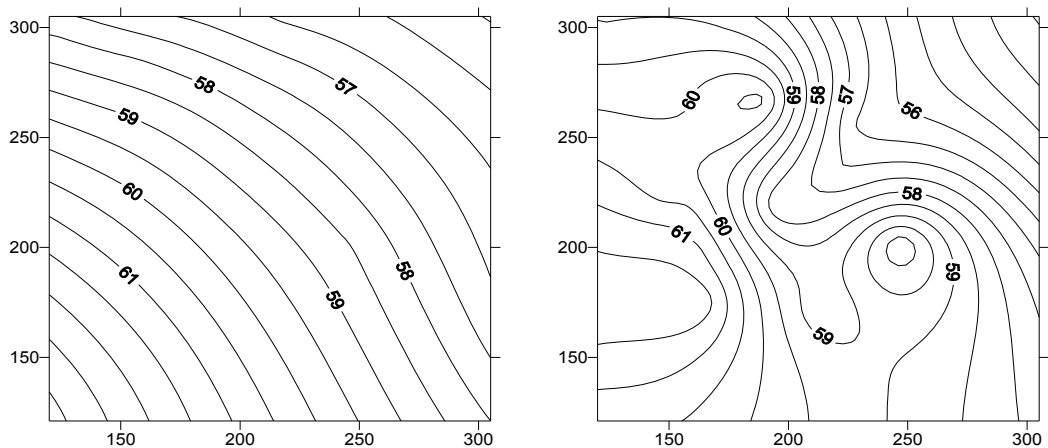

Figure 5: SPL maps (30 samples input without external drift on the left and with drift on the right). 

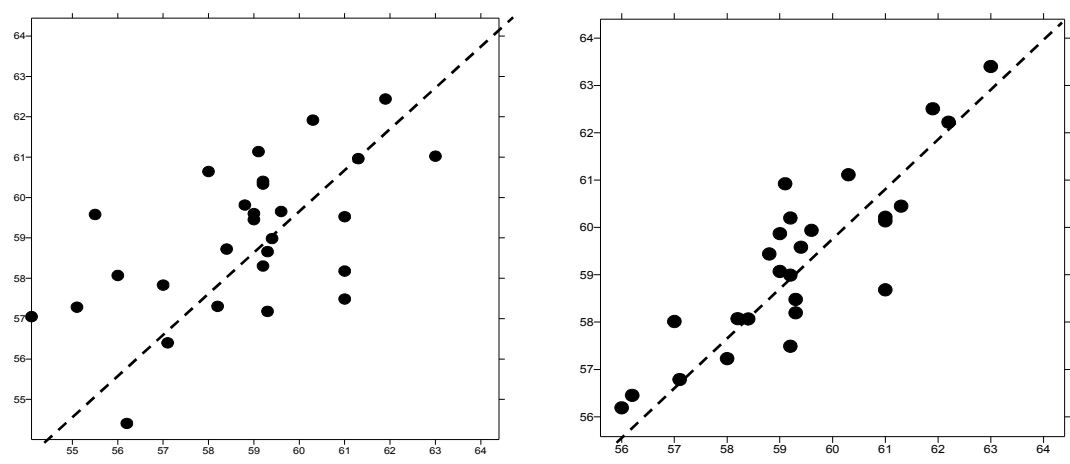

Figure 6: Scatterplot sampled values vs estimated (30 samples input without drift).

In the following table 2 parameters of each scenario are shown.

Table 2: Generalized covariance parameters for each case.

\begin{tabular}{|c|c|c|c|c|c|}
\hline $\begin{array}{c}\# \\
\text { samples }\end{array}$ & $\begin{array}{c}\text { Ext. } \\
\text { drift }\end{array}$ & K order & Co $_{0}$ & bo & b1 \\
\hline 15 & No & 1 & $2.1710^{-5}$ & - & - \\
\hline 15 & Yes & 1 & $8.9010^{-6}$ & - & $1.410^{-12}$ \\
\hline 30 & No & 1 & $1.2110^{-5}$ & - & $1.0810^{-10}$ \\
\hline 30 & Yes & 1 & - & $2.0710^{-5}$ & - \\
\hline
\end{tabular}

Lastly, with reference to the data estimated for each of the 4 scenarios, table 3 indicates the values obtained from the linear correlation $\mathrm{R}$, from the mean error, and from the mean squared error. The bottom row shows the same parameters according with the ISO model.

Table 3: Quantitative analysis for each scenario.

\begin{tabular}{|c|c|c|c|c|}
\hline \# samples & External drift & $\mathbf{R}$ & $\begin{array}{c}\text { Mean } \\
\text { error }\end{array}$ & $\begin{array}{c}\text { Mean } \\
\text { Sqrt. error }\end{array}$ \\
\hline 15 & No & 0.13 & -0.15 & 3.13 \\
\hline 15 & Yes & 0.44 & -0.23 & 2.11 \\
\hline 30 & No & 0.41 & -0.10 & 1.81 \\
\hline 30 & Yes & 0.76 & -0.04 & 1.26 \\
\hline ISO Mod & & 0.73 & 1.54 & 1.95 \\
\hline
\end{tabular}




\section{Discussion and conclusion}

The comparison of the acoustic maps allows a number of considerations to be made. Firstly, it should be noticed that in the case study of Intrinsic Kriging without drift, especially for a subset with a limited number of measurements, the application returns an estimate with low variability on a small scale in which the generalised covariance constitutes the only nugget effect. This, however, prevents local effects from being perceived. The introduction of drift, on the other hand, represents an improvement in this regard. It is possible to appreciate the single contributions of the two components which are combined to produce to the final estimate; on the one hand the data derived from the sample and the auxiliary information from the model on the other hand. The way in which the external drift contributes to the estimate may be seen in figures 3 and 5 . In both cases in fact a trend is seen which recalls the variability structure of the phenomenon and is the result to the integration of the auxiliary information provided by the model with the sampled data. For a comparison one should note the map in figure 2 which shows the acoustic climate obtained by means of the use of the ISO model. Moreover, the model utilised in the case study, as may be observed in the same figure 2 (right side) is characterised by a generalised underestimation of the sound pressure values. This is because it was calculated from the noise emission specifications provided by the machinery manufacturers and without carrying out any phonometric measurements in the field. Such an omission, however, does not affect the quality of the estimate as the calibration of the variable being studied is guaranteed by the information provided by the sampling, which also allows some small scale fluctuations due to local effects to be perceived. By and large, it is possible to affirm that, all inputs being equal, the introduction of external drift contributes to an improvement in the quality of the estimate, and may be observed both from the synthetic indicators in table 3 and from the relative scatterplots in figures 4 and 6 . As evidence of this, it may be noted in table 3 that the correlation index increases with the reduction of mean error and estimate variation. In this regard, the greater density of the points along the diagonal in scatterplot with drift (figures 4 and 6 on the right) goes to qualitatively confirm this tendency. A further point for consideration is a comparison of the data relative to the estimate using 15 measurements and drift, with respect to those using all 30 measurements without the auxiliary help of the model (see table 3 rows 2 and 3). The data show that a drastic reduction in the number of sampling measurements in the input (in this case equal to $50 \%$ of the total) is not translated into a drastic reduction in the quality of the estimate, which on the contrary, according to the correlation factor and the general information given in table 3 , remains more or less unchanged.

\section{References}

[1] Alfaro Degan G, Lippiello D, Pinzari M. (2009). Occupational health and safety management systems: comparison between BS OHSAS 18001: 2007 and Italian Decree 81/2008. WIT Transactions on Biomedicine and Health, 
Vol. 14, WIT Press, 2009, ISSN: 1743-3525, pp. 401-409, doi: 10.2495/EHR090391.

[2] Dowd P.A., Li S. Knowledge and geographical information-based system for noise impact assessment of surface mining and quarrying projects. Transactions of the institution of mining and metallurgy Section A-Mining Industry, Vol. 109, pp. A1-A13, 2000.

[3] Nanda, S.K., Tripathy D.P., Patra S. K. Fuzzy inference system-based noise prediction models for opencast mines. International Journal of mining reclamation and environment. 23 (4), pp. 242-260, 2009.

[4] Alfaro Degan G, Lippiello D, Pinzari M. A geostatistical approach to the functional analysis space technique: a case of study. Advances in Safety and Reliability - Proceedings of 16th European Safety and Reliability Conference (ESREL). Ed: Kolowrocki K, Taylor \& Francis, Vol. 1, pp. 4552, 2005.

[5] Alfaro Degan G, Lippiello D, Lorenzetti S, Pinzari M. Vibration assessing models: comparison between methods. WIT Transactions on Biomedicine and Health, Vol. 16, WIT Press, 2013, ISSN 1743-3525, pp. 59-69, doi. 10.2495/EHR130061.

[6] Cinar, I. and Sensogut, C. Evaluation of environmental factors affecting noise propagation. Environmental Monitoring, 153 (1-4), pp. 377-382, 2009.

[7] De Melo Neto R. P., Kohlman Rabbani E.R., Barkolebas Junior B and Lago D.M.G. Quantitative noise analysis at two marble finishing plants in Olinda, Pernambuco, Brazil. Work, 41 (suppl 1), pp. 5850-5852, 2012.

[8] Alfaro Degan G, Lippiello D, Lorenzetti S, Multari F, Pinzari M .Combined evaluation of the noise and vibration at a travertino quarry. Recent Developments in Acoustics, Noise and Vibration. Proceedings of 16th International Congress on Sound and Vibration (ICSV16). Eds. M. Pawelczyk \& D. Bismorvol, IIAV: Krakow, vol. 3, pp. 1940-1947, 2009.

[9] Ahmed S, de Marsily G. Comparison of geostatistical methods for estimating transmissivity using data on transmissivity and specific capacity. Water Resources Research, 23, pp 1717-1737, 1987.

[10] Galli, A., Meunier, G. Study of a gas reservoir using the external drift method. In: Matheron, G., Armstrong, M. (Eds) Geostatistical case studies. Reidel, Hingham, Mass, pp. 105-120, 1987.

[11] Hudson G, Wackernagel $\mathrm{H}$. Mapping temperature using kriging with external drift: theory and example from Scotland. International Journal of Climatology, 14(1), pp. 77-91, 1994.

[12] Baume O; Gauvreau, B; Berengier, M; Junker, F ; Wackernagel, H; Chiles, JP. Geostatistical modeling of sound propagation: Principles and a field application experiment. Journal of the Acoustical Society of America. 126 (6), pp. 2894-2904, 2009.

[13] Genikhovich E, Filatova E, Ziv, A. A method for mapping the air pollution in cities with the combined use of measured and calculated concentrations. International Journal of Environmental Pollution, 18(1), pp. 56-63, 2002. 
[14] Desbarats AJ, Logan CE, Hinton MJ, Sharpe DR (2002) On the kriging of water table elevations using collateral information from a digital elevation model. Journal of Hydrology, 255 (1-4), pp. 25-38, 2002.

[15] Matheron, G. The Intrinsic Random Functions and their applications. Advances in Applied Probability, 5, pp. 439-468, 1973.

[16] Rao Radhakrishna, C. Estimation of variance and covariance components. MINQUE Theory. Journal of multivariate analysis, 1 (4), pp. 257-275, 1971. 